\title{
Study on Polysemy From the Perspective on Cognitive Metonymy*
}

\author{
WANG Xian-ming \\ Qufu Normal University, Qufu, China
}

\begin{abstract}
The more common the world, the more meanings it has. We can say that polysemy is the role and monosemy is the exception. Polysemy is a common phenomenon for students in language learning, but is also the difficulty of their learning. Studying semantic changes leading to the growth of polysemy is an effective way in the research of wording-building. More importantly, the formation of polysemy is closely related to culture and human activities, and especially in the cognitive domains of human activities. As a primary means, metonymy plays a key role in the formation from one word sense to another extension. Therefore, from the perspective of cognitive metonymy, for teachers, to let the students know the network of meaning-building and the relationships of different elements in word-building is extremely important; and for students, to know the rule for the formation of polysemy has essential implications for their vocabulary acquisition.
\end{abstract}

Keywords: polysemy, cognition, metonymy, vocabulary

\section{Introduction}

Polysemy is an important part of English vocabulary, so linguists pay close attention to its formation, development, and the relationship among the different meanings of the words. But the traditional research only focuses on the internal structure of the words and ignores the external factors, such as the impact of human cognitive activities and life experience on the lexical meanings, and therefore, substantive research of polysemy has not been researched (Woods, 1996). With the development of cognitive linguistics, scholars turn the research direction of linguistic phenomena to this field, so polysemy research is paid wide attention. Cognitive linguistics combines language structure with human activities, and the structure of thinking, which considers language, is a part of human cognition and in other areas, its formation and development and human cognition are indivisible, including social, cultural, and psychological structure and other factors, therefore, a profound understanding of language cannot be inseparable from the interdisciplinary research (Altarriba \& Mathis, 1997). The structure of language based on human experience, which is the concept in the process of knowing the external world.

The theoretical model of category in cognitive linguistics provides support for the interpretation of the relationship among different meanings of polysemy. Polysemous meanings are produced in order to meet the

\footnotetext{
* This paper is the stage research result of "A Brief Study of NP Anaphora in Sports English Texts From the Perspective of Cognitive Metonymy" (No. 10DWXJ08) -2010 Shandong Social Science Research Project.

WANG Xian-ming, lecturer, College English Department, Qufu Normal University.
} 
needs of people when they want to understand and express the new meaning of the words which are with old expressions. A word with the expression of social and human development will produce different meanings, which constitute the word semantic category, in which each item among the statuses is not in equal degree, because there are the division of the typical members and peripheral members. The prototype category theory provides theoretical foundation for the cognitive study of polysemy, which also shows that language is formed in the process of human understanding of the world, reflecting the law of knowing the world. Cognitive research shows that people tend to know more specific things, and then know new things through the projection from concrete domain to the abstract domain.

In the process of language use, many basic abstract concepts, such as time, place, action, state, etc., such words are evolved through metaphor or metonymy mode of people thinking. Polysemy is a reflection of human cognition, while the cognitive mechanism of metaphor and metonymy plays an important role in the formation process of semantic category (Garton \& Richards, 2008). This paper analyzes the generation process of polysemy from the cognitive perspective of metonymy, and researches semantic generation mechanism of polysemous words.

\section{Cognitive Metonymy and Polysemous Semantic Category}

\section{Study of Cognitive Metonymy}

In recent years, research on metonymy shows that it is not only a rhetorical phenomenon, but also an important way of cognition and thinking, and study of the motivation of metonymy in language is gradually prevailed. Kroll and Tokowicz (2001) thought:

Metonymy is a conceptual category, the source domain mapping to the target domain, which belongs to the same functional category (or idealized cognitive model). They are connected by a pragmatic function; the target domain can be activated in the psychological source domain. (pp. 49-71)

One of the basic functions of metonymy is substitute function, which is not simply a thing instead of another, but a combination of both to form a complex new meaning. Cognitive model is expressed as "X plus Y".

Idealized cognitive model provides the frame foundation for the study of metonymy. It can be defined as an ordered cognitive structure, in which the experience and human behavior are highly generalized, so as to provide concise and ideal cognitive framework for people to know the world. In other words, they provide a denotation of language and behavior of human, telling us what the world is like, and how to communication with other people (Roelofs, 2000).

We see this sentence, "Bush bombed Iraq". In fact, the meaning of the sentence is not Bush bombed Iraq. We get this meaning, because according to experience, the one who is in control should be responsible for the accused person's behavior, while Bush is the ruler of the United States Army, so we think he should be responsible for his own troops and their bombing behavior.

For category - member model, research shows that member is the most typically used to refer to the whole category (El-Okda, 2005; Macalister, 2010). For instance, for the category of mother, mother as a housewife in our cultures is a better representative, which comes from the metonymic thinking, and self-category has the most typical characteristic of this category. According to the idealized cognitive model, metonymy can be divided into 
two kinds: One kind exists between the whole and the parts in idealized cognitive model, in which we can understand the parts well and vice versa; another kind lies in between the parts of the same model, through a part to understand another part. By metonymy, center meanings of words expand to other areas, which form new meanings of words.

\section{Cognitive Metonymy and Polysemy Formation}

Metonymy is an important resource in word formation. Taylor (1989, p. 202) with the metonymy resolution of semantic extension summarized it into the following several aspects: different prominent entities, perspective of an integral part, and a perspective implied meaning. There are many examples through the metonymic extension to a part of the overall perspective of the conceptual structure to realize polysemy. We take "hand" as an example; it is often used to refer to the whole of metonymy, this conceptual metonymy is called "The Hand Stands for the Person", such as "They are mostly factory hands". In this sentence, "hand" can also be used to refer to the general ability, expertise, an industry or occupation skills, such as "skilled hand", "good hand", and so on. "Hand" is closely related to the right and the controlling force, which usually refers to the skills, methods, and strategies, thereby creating a "The Hand Stands for Control" and "Controlis Holding in the Hand" metonymic relation, so, we can use the "The firm is at his hands" to express the meaning of the company is in the charge of him.

Many of the English vocabulary classification is more complex. Many words have both a noun meaning and a verb meaning. The important reason of this phenomenon is closely related to the cognitive metonymy. In the noun-verb transition, concepts represented by noun and verb belong to the part and the whole relationship, so the conversion between them is part of a whole generation of metonymy. Relevant examples include: to butcher the cow, to parent the children (agent refers to the generation of action), to summer in Japan, to honeymoon in HongKong (time refers to the generation of action), to fool the man, to copy the paper (results refer to action), etc.

Another phenomenon is about some words related to human body which can be a very good interpretation that the metonymic mechanism plays an important role in the process of the formation of the some lexical polysemy. The basic meaning of the word "head" is the head of the human body, but the word can also be used to refer to the concept of the human, such as "It costs about a head to eat here for $\$ 10$ " (eating here everyone should pay US \$10); the word can also refer to a person's intelligence and abilities, such as "a good head for something" which refers to the meaning of good or well-done. In addition, "head" can also refer to the summit, leaders and the title of an article, in which the significance and metonymy is inseparable, because the human head is the core part of the whole body, controling the normal operation of the body. When we talk about someone, we will first think of his facial features of this person, so in the basis of cognitive metonymy, through the metaphor, we can extend the derived meaning. See the word "eye", whose meaning includes all the physiological component of organs, including the nature and function of organs, such as eyes, visual acuity, and observation, as in the sentence "He has a quick eye for mistakes" (he could tell the error at a glance), the meaning of eyes is about its function, that is, observation.

\section{Teaching Strategies in Polysemy}

Through the above analysis, we can see that the formation and development of cognitive metonymy plays an important role in word sense, which can help us better understand the meaning of polysemy. To explain the 
emergence and development of polysemy from the cognitive perspective, we can help students better understand the relationship between the different senses of polysemous words, the meaning extension rules, so as to improve the efficiency of vocabulary acquisition, because learning flexibly is much easier than blindly memorizing.

Polysemy is the result of human cognitive abilities, and also for the needs of language economy principle (Borg, 2003). For a word originally, there is a single meaning, gradually it gains new meaning in the language development process, according to the prototype category theory, the center or core meaning of a polysemous word in semantic category is of the significance of the prototype, and other meanings are further extended in this foundation (Holliday, 1994). Teachers should help students to construct the sense network of a polysemous words in teaching, let students understand the relationship between the core meaning of the polysemy and the non-core meaning and secondary meaning, and let students learn how to derive some non-core senses according to the core meaning, which can lighten the burden of students' learning.

To establish a network of word sense, it requires students to understand the concept of the importance of vocabulary, but teachers also need to cultivate students' cognitive ability (Nation \& Macalister, 2010), besides, in the process of teaching, teachers should also help students expand the knowledge system. Cognitive linguistics is encyclopedic semantics. Semantic is a complete system, but also can be decomposed. To better understand semantics, learners need to establish a complete related concept in a knowledge system framework. The knowledge framework helps trigger any node on the frame to activate knowledge. When we meet a word, a series of related objects, scene or social knowledge will quickly appear in our mind. Learning foreign language vocabulary, learners' conceptual process is more complicated (JIANG, 2000, 2002), because the target language and the encyclopedia of knowledge system in the mother tongue is not exactly the same, and thus it will cause the obstacles in the process of acquisition. For example, the word "face" in English and in Chinese can be basically identical, but they still have some differences in usage. Even in English, there is the expression of "lose one's face (shameful)". In most cases, "face" shows external appearances and emotional meaning, while the meaning of Chinese "face" is not the same. When Chinese say "face", they refer to a personal friendship and face reflects the feelings among people, to adjust the psychological tendency of individuals, and to maintain the normal relationship of people in society, which also reflects the cultural difference in different languages.

\section{Conclusions}

Semantic extension needs metonymy cognitive structure, in which we can get the extension for the cognitive metonymy words from the center meaning to non-centered sense. The meaning extension process is based on the conceptualism, inseparable from other social and cultural knowledge, so the teacher in the teaching process should pay attention to the cultivation of students' metonymy thinking ability, and the ability to realize the importance of other relevant social and cultural knowledge.

\section{References}

Altarriba, J., \& Mathis, K. M. (1997). Conceptual and lexical development in second language acquisition. Journal of Memory and Language, 36, 550-568.

Borg, S. (2003). Teacher cognition in language teaching: A review of research on what languageteachers think, know, believe, and do. Language Teaching, 36(2), 81-109. 
El-Okda, M. (2005). EFL student teachers' cognition about reading instruction. The Reading Matrix, 5(2), 43-60.

Garton, S., \& Richards, K. (Eds.). (2008). Professional encounters in TESOL: Discourses of teachers in teaching. Basingstoke: Palgrave Macmillan.

Holliday, A. (1994). Appropriate methodology and social context. Cambridge: Cambridge University Press.

JIANG, N. (2000). Lexical representation and development in a second language. Applied Linguistics, 21, 47-77.

JIANG, N. (2002). Form-meaning mapping in vocabulary acquisition in a second language. Studies in Second Language Acquisition, 24, 617-637.

Kroll, J. F., \& Tokowicz, N. (2001). The development of conceptual representation for words in a second language. In J. L. Nicol, \& T. Langendoen (Eds.), One mind, two languages: Bilingual language processing (pp. 49-71). Cambridge, M.A.: Blackwell.

Macalister, J. (2010). Investigating teacher attitudes to extensive reading practices in higher education: Why isn't everyone doing it? RELC Journal, 41(1), 59-75.

Mann, S. (2011). A critical review of qualitative interviews in applied linguistics. Applied Linguistics, 32(1), 6-24.

Nation, I. S. P., \& Macalister, J. (2010). Language curriculum design. New York and London: Routledge/Taylor and Francis.

Roelofs, A. (2000). Word meanings and concepts: What do the findings from aphasia and language specificity really say?. Bilingualism: Language and Cognition, 3, 25-27.

Taylor, S. (1989). Semantics: An introduction to the culture of meaning (p. 202). Oxford: Basil Blackwell.

Woods, D. (1996). Teacher cognition in language teaching: Beliefs, decision-making and classroom practice. Cambridge: Cambridge University Press. 